\title{
THE NATURE OF THE COMPLEXES FORMED BETWEEN SODIUM AND TELLURIUM IN LIQUID AMMONIA
}

\author{
By Charles A. Kraus and C. Y. Chiu \\ Received June 27, 1922
}

\section{Introduction}

The fact that liquid ammonia is capable of dissolving the alkali metals, which, in the absence of moisture and other impurities, may be recovered upon evaporation of the solvent, was discovered by Weyl. ${ }^{1}$ These solutions have since been the subject of extended study by various investigators. The physical properties of the alkali metal solutions in ammonia are now fairly well understood, but the chemical properties of these solutions have thus far received comparatively little attention. From the nature of the elements, it may be inferred that these solutions will prove to be very reactive chemically. Indeed, it has been found that at the boiling point of liquid ammonia the alkali metals react with many nonmetallic elements to form salts, while with the salts of less electropositive metals they react with the replacement of the electropositive constituents. Of especial interest, however, are the reactions in which the alkali metals combine directly with various less electropositive metallic elements.

Joannis ${ }^{2}$ observed that mercury, lead, antimony and bismuth react with the alkali metals in ammonia solution. To the compounds formed with sodium he assigns the formulas $\mathrm{NaHg}_{4}, \mathrm{NaPb}_{2}, \mathrm{Na}_{3} \mathrm{~Pb}, \mathrm{Na}_{3} \mathrm{Bi}$ and $\mathrm{Na}_{3} \mathrm{Sb}$. It has since been shown that the alkali metals also react with selenium, tellurium, tin, arsenic, phosphorus, etc. It has further developed that the reactions are not as simple as Joannis assumed, since apparently without exception, when reaction occurs between an alkali metal and a less electropositive element in ammonia solution, a series of compounds is formed, the solubilities of which in liquid ammonia may differ very largely. As a rule, the first compound formed, in which the proportion of the alkali metal is highest, is less soluble in liquid amrnonia than the succeeding compounds in which the relative amount of the more electronegative element is greater. The reactions are, in general, accompanied by striking color changes which presumably are due to the presence in the solution of certain definite complexes.

The solutions formed in ammonia by reaction between sodium and lead have been studied by Smyth. ${ }^{3}$ It has been shown that these solutions are electrolytic in nature." Upon electrolyzing a solution of the sodium-lead complex in equilibrium with metallic lead, Smyth found that 2.26 gram atoms of lead are deposited on the anode per equivalent of ellectricity passing through the solution. This shows that lead is associated with a negative ion in these solutions. At the same time, the fact that the atomic ratio is not an integer indicates that a mixture of complexes is present. In the case of lear, the ratio of sodium to lead in the solution is practically independent of the concentration.

\footnotetext{
1 Weyl, Ann. Phys., 121, 601 (1864).

${ }^{2}$ Joannis, Compt. rend., 113, 795 (1891); 114, 585 (1892).

${ }^{3}$ Smyth, This Journal, 39, 1299 (1917).

4 Kraus, ibid, , 29, 1563 (1907); 43, 758 (1921).
} 
The reactions between sodium and antimony have been similarly investigated in this Iaboratory by Peck. The atomic ratio of antimony to sodium in a solution in equilibrium with antimony was found to vary, as a function of the concentration, between 1.5 and 2.3 .

The present investigation was undertaken for the purpose of studying the reaction between sodium and tellurium. This system has the advantage over others in that the rate of reaction is extremely rapid, thus eliminating the possible loss of sodium due to the formation of sodium amide. At the same time, the color changes accompanying the reaction are comparatively sharp.

This reaction has been partially investigated by $\mathrm{Hugot}^{6}$ who found that the first product of the reaction is a colorless gelatinous precipitate insoluble in liquid ammonia, to which he assigns the composition $\mathrm{Na}_{2} \mathrm{Te}$. Upon addition of more tellurium, this compound goes into solution. The resulting solution, according to Hugot, has the composition $\mathrm{Na}_{2} \mathrm{Te}_{3}$. These results of Hugot will be discussed somewhat more in detail below.

The atomic ratio of sodium to tellurium in liquid ammonia has also been studied by Allison ${ }^{7}$ and later by Power. ${ }^{8}$ According to these investigators, the ratio of tellurium to sodium varies as a function of concentration, exhibiting a maximum value of approximately 1.95. The results of Allison and of Power are somewhat in doubt owing to the fact that the tellurium employed was not specially purified.

The equilibrium between the normal telluride $\mathrm{Na}_{2} \mathrm{Te}$ and tellurium in water has been investigated by Tibbals, ${ }^{2}$ who found that the average composition of the solution corresponds to $\mathrm{Na}_{4} \mathrm{Te}_{3}$; the solution upon evaporation to dryness decomposes into tellurium and normal sodium telluride. Tibbals apparently has failed throughout his work to take account of the fact that solutions of $\mathrm{Na}_{2} \mathrm{Te}$ in water, as well as solutions of the complex telluride, are hydrolyzed to a considerable extent. The slight solubility and the relative instability of hydrogen telluride in water might be expected to influence his results appreciably.

Pellini and Guercigh ${ }^{10}$ have studied the system sodium-tellurium by means of thermal analysis. They established the existence of the compounds $\mathrm{Na}_{2} \mathrm{Te}_{2} \mathrm{Na}_{3} \mathrm{Te}_{2}$ and $\mathrm{Na}_{3} \mathrm{Te}_{7}$, but could not detect the presence of the compounds $\mathrm{Na}_{2} \mathrm{Te}_{3} \mathrm{Na}_{2} \mathrm{Te}_{4}$, and $\mathrm{Na}_{2} \mathrm{Te}_{5}$. The behavior of this system appears to differ markedly from that, of sodium and selenium which forms an unbroken series of compounds having the formula $\mathrm{NaSe}_{n}$, with $n$ ranging from 1 to 6 .

It is of interest to note that of the various methods employed that of thermal analysis gives the highest ratio of tellurium to sodium. We shall see below that in ammonia solution the highest ratio in which the elements occur differs but little from the highest ratio found from thermal analysis.

5 Peck, This Journal, 40, 335 (1918).

- Hugot, Compt. rend., 129, 299 and 388 (1899).

${ }^{7}$ V. C. Allison, Thesis, Clark University (1916).

${ }^{8}$ F. W. Power, ibid., Clark University (1917).

- Tibbals, This Journal, 31, 902 (1909).

${ }^{10}$ Pellini and Guercigh, Atti. accad. Lincei, [5] 19, II, 350-56 (1910); Zentr, 81, II, 1648 (1910). 


\section{Purification of the Materials}

Ammonia.-Ordinary liquid ammonia from a large cylinder was transferred into a small tank to which about $10 \mathrm{~g}$. of sodium had previously been added. The ammonia was distilled over into the reaction tube by cooling the latter in a bath of boiling ammonia.

Sodium.-The method of preparing pure sodium was practically the same as that used by Peck.

The sodium was cut and introduced into a weighed glass tube which was constricted to at capillary at a point several $\mathrm{cm}$. above the lower end which was closed, thus forming a small capsule. On the top of the sodium was placed a glass rod about $8 \mathrm{~cm}$. long and having a diameter slightly less than that of the glass tube. This glass weight was intended to assist in breaking the oxide film when melting. The glass tube was joined to a mercury vapor pump by means of de Khotinsky cement and evacuated. It was then heated in a paraffin bath. When the sodium was melted, ammonia was introduced. This forced the molten sodium through the capillary tube into the capsule underneath, leaving the oxide behind. The capsule was then sealed off at the capillary. The capsule was cleaned by warming, wiping with a piece of paper, and finally with a piece of cloth soaked with alcohol. The sodium oxide was removed from the remaining portion of the tube by adding alcohol and water in drops. After having been cleaned and dried, the capsule, together with the glass tube, was again weighed. The difference in weight gave the weight of sodium in the capsule.

Tellurium.-As is well known, ordinary tellurium contains impurities, chiefly selenium, whose separation from tellurium is often attended with considerable difficulty. After many trials, the following procedure was adopted; it is a modification of that of Norris, Fay and Edgerly. ${ }^{11}$

Tellurium powder was first melted in a vacuum. After breaking up the resulting mass, it was placed in a porcelain boat, which was then pushed into a Pyrex glass tube, in a furnace of the type used for combustions in which it was finally distilled. Before heating, the air was displaced by a current of ammonia which was allowed to escape through a mercury trap, and which was maintained throughout distillation. The golden-yellow vapor of tellurium condensed on the glass tube near the end of the boat, forming mercury-like globules, which coagulated to a large mass. Further in the tube, leaf-like crystals were formed, somewhat resembling iodine crystals. When most of the tellurium in the boat was gone, distillation was discontinued. In this operation heavy metal tellurides and silicates were left behind in the boat.

The tellurium was then dissolved in hot dil. nitric acid and the solution rapidly evaporated. The residue was ignited and dissolved in conc. hydrochloric acid and sulfur dioxide was passed into the solution until the red precipitate was superseded by a quantity of black precipitate. This was filtered off. In this process most of the selenium was removed. To the filtrate a volume of water about twice that of the original solution was then added, the solution heated to boiling and sulfur dioxide was passed in until the solution became colorless on vigorous stirring. The precipitate formed under these conditions settled well and could be filtered readily. The supernatant liquid was decanted and water added. This same process was repeated several times, and finally the residue was filtered by means of a suction pump and washed.

The precipitated tellurium was again dissolved in cold nitric acid (sp. gr, 1.25), about $10 \mathrm{~g}$, in $300 \mathrm{cc}$. of acid. If any precipitate separated, it was filtered off. To

${ }^{11}$ Norris, Fay and Edgerly, Am. Chem. J., 23, 105 (1900). 
the solution, conc. nitric acid was added. This served to increase the size of the crystals later formed. The solution was evaporated in an evaporating dish covered with a watch glass on a water-bath kept at temperatures between $80^{\circ}$ and $85^{\circ}$. Rapid evaporation, either by raising the temperature of the bath or by passing a current of air over the surface of the solution, causes imperfect crystallization. The solution has great tendency to supersaturate; hence, seeding is helpful. When $90 \%$ of the liquid had evaporated, the dish was allowed to cool, and the remaining liquid was drained off. The crystals were then washed with dil. nitric acid and recrystallized. The metal was precipitated by means of sulfur dioxide and washed until free from chloride and sulfate. The powder was then dried and melted in a vacuum.

The tellurium in this form was ready for casting into sticks. It was placed in a Pyrex glass tube of desired size, connected to the vacuum line and evacuated. The lower end of the tube was heated by direct flame. When all the tellurium was melted, the vacuum line was shut off, and ammonia gas was introduced. This was intended to collapse the gas bubbles present on the walls of the tube. During cooling, the upper part of the stick was heated, as tellurium contracts greatly upon solidifying, thus leaving a cavity in the top of the stick, unless the procedure mentioned is employed. If the stick failed to slip out of the glass tube, the part of the tube containing tellurium was scratched with a file, warmed in a flame and immediately immersed in cold water. After having freed the stick from the glass tube, it was shaved down and the upper part was usually sawed off. Near the lower end a hole was drilled, through which a wire was passed for the purpose of suspending the stick in the reaction tube.

\section{Apparatus}

The apparatus is shown in outline in the accompanying figure.

$A$ is the reaction tube which is provided with two arms $B$ and $C$. The whole is placed in a Dewar tube, $D$, and held in position by means of a rubber stopper $E . F$ and $G$ are two side arms attached to $B$ and $C$ respectively. These are joined together at $H$ to another tube $K$. $L$ is a rubber stopper, through which passes the tube $M$. $M$ is connected to tube $O$ by the rubber tube $N$. This device permits the long tube $P$, on which the tellurium stick hangs, to be raised or lowered. The tube $Q$, which connects with the ammonia tank, is provided with a similar device. $S$ is a stopcock.

\section{Procedure}

The experimental method used in this work consists in dissolving a known weight of sodium in liquid ammonia and determining the weight of tellurium dissolved during different stages of the reaction. It is obvious that no air or moisture should be allowed to enter the reaction tube. When the reaction tube has been thoroughly cleaned and dried, it is placed in position as shown in the figure. The tube $Q$ is lowered until the lower end almost touches the bottom of the reaction tube. The weighed tellurium stick is hung on $\mathrm{H}$. Then $Q$ is connected to the ammonia tank and to the mercury trap. The joints with the rubber tubes at $N$ and $J$ are made tight by tying with copper wire. A slow current of ammonia 
from the tank is forced through the apparatus until all the air has been displaced. Then the Dewar tube is filled about half full with liquid ammonia. The ammonia from the small supply tank condenses readily when the height of the mercury in the trap is about $15 \mathrm{~cm}$. When the desired amount of ammonia has been condensed, the stopper $L$ carrying the tellurium is momentarily raised, and the capsule with the known weight of sodium is dropped in TO $\mathrm{NH}_{\text {TAN }}$ TA after having been previously broken by scratching with a file and touching with hot glass. This is always done with the help of an assistant. The ammonia supply tank is then turned off. When all the sodium is dissolved, the tellurium stick is lowered until it is half immersed in the solution; and at the proper stage it is raised. The Dewar tube is then filled with ammonia to a point at least as high as the top of the stick. To wash the stick free from solution, ammonia from the tank is condensed on the side of tube $B$. This can be done easily and efficiently if $B$ is not too large and if the whole tube is given a rotatory motion so that the stick touches the walls of tube $B$. When the washing is completed, the stick is taken out, and in place of stopper $\mathrm{L}$ another stopper is immediately inserted. The stick is then quickly dried by means of a water jet purmp and again weighed. Thus the composition of the solution at each

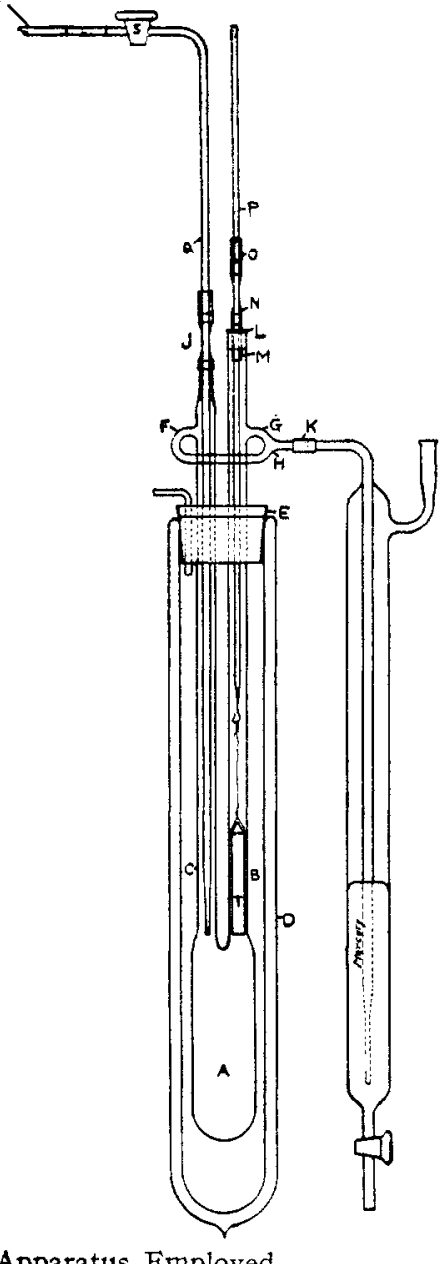
stage of the reaction is known. Whenever the stick is lowered, raised, or taken out, a vigorous current of ammonia must be maintained to prevent the entrance of air.

It is well known that concentrated solutions of sodium in ammonia are golden-red while dilute solutions are blue. As soon as the stick is lowered into the metal solution, a white precipitate is formed and the solution, after a time, assumes a greenish tint. The compound formed is distinctly 
crystalline. The reaction is very vigorous, especially when the solution of sodium is concentrated. As the white precipitate is formed in increasing quantity, the green color of the solution becomes more pronounced. It then changes to yellowish-green and becomes transparent. The stick is then raised and washed, while the solution is stirred either by lowering $Q$ and running through a small current of ammonia, or by removing the reaction tube from the bath and warming the bottom with the bare hand. If the solution is still green, the stick is again lowered into the solution. This process is repeated until the solution is just light yellow. This point indicates that all free sodium has disappeared. The stick is then withdrawn from the apparatus, weighed, and replaced in the reaction tube.

The white precipitate which, as will be shown below, consists of the normal telluride, $\mathrm{Na}_{2} \mathrm{Te}$, is somewhat soluble in liquid ammonia. In the presence of excess tellurium, the normal telluride reacts rapidly with the metal forming a very soluble higher telluride. This second stage of the reaction is allowed to proceed until the original precipitate formed is just on the point of disappearing. Under these conditions the solution is in equilibrium with the normal telluride and the composition of the solution is fixed. At this point the stick is again withdrawn from the solution, washed, and finally removed from the reaction tube and weighed. After weighing, it is again returned to the reaction tube. On again introducing the stick into the solution, further reaction takes place between the tellurium and the telluride already in solution. This reaction is allowed to proceed until the solution is in equilibrium with the free element. The tellurium dissolved is then finally obtained after washing the stick and weighing.

The rate of reaction depends, of course, upon the surface of solid tellurium exposed to the solution and on the frequency with which the solution is stirred. The following results will be of interest in showing how the final equilibrium is reached.

G.

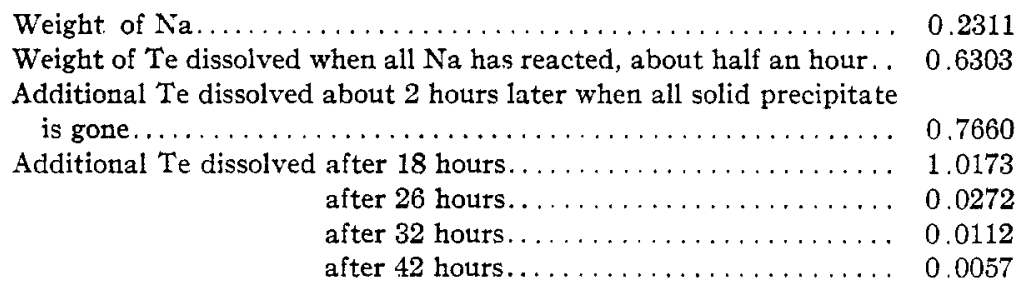

The reaction is accompanied by a series of striking color changes. The yellow solution first turns pale brown, then greenish and gray. These colors, however, are only transitory, the solution becoming blue and transparent, and then violet-purple, resembling a strong solution of potassium permanganate. The final color of the solution is invariably deep red. 
The change from purple to red occurs when the normal telluride has just disappeared. The solutions are exceedingly viscous when concentrated.

\section{Experimental Results}

The results obtained are given in the following table. $A$ is the point at which the free metallic sodium in solution has just disappeared, the solution being colored pale yellow; $B$ is the point at which the initial insoluble precipitate formed has just gone into solution, in other words this is the point at which the solution is in equilibrium with the normal telluride; and $C$ is the point at which the solution is in equilibrium with solid tellurium. In the table, the concentrations are expressed in gram atoms of sodium per thousand grams of ammonia. The density of liquid ammonia at its boiling point is 0.674 , from which the true concentration of the solutions may be approximately determined, assuming that the volume of the solution is equal to that of the pure ammonia present. The amount of sodium used varied between 0.04 and $0.8 \mathrm{~g}$. and that of ammonia between 10 and $21 \mathrm{~g}$.

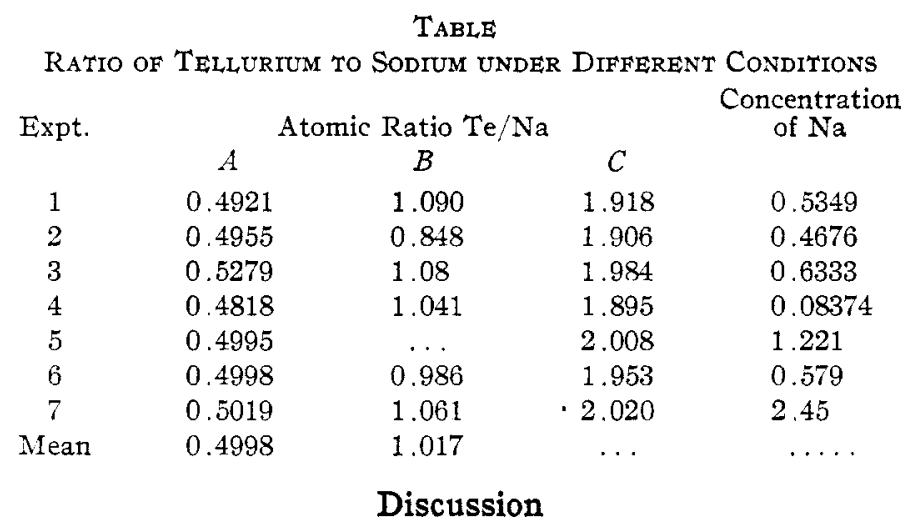

From an inspection of the table, it is clear that the initial precipitate formed is the normal telluride, $\mathrm{Na}_{2} \mathrm{Te}$. The mean value obtained for the ratio of sodium to tellurium in the initial compound according to the experiments is 0.4998 , which corresponds almost precisely with the composition of the normal telluride. As has already been mentioned, this telluride is appreciably soluble and precipitates from solution in crystalline form. This is somewhat at variance with the results previously obtained by Hugot, who states that the precipitate is gelatinous in nature. Hugot does not describe his experiments in sufficient detail to make it possible to determine precisely the cause of this discrepancy. It is not improbable, however, that traces of moisture may have been present in his ammonia, as a consequence of which a gelatinous precipitate of hydroxide would naturally have resulted. At the point $B$, at which the solution is in equilibrium with the normal telluride, the mean value found for the ratio of 
tellurium to sodium is 1.017 . This corresponds very nearly with the formula $\mathrm{Na}_{2} \mathrm{Te}_{2}$. The variation of the ratio from the value 1.0 is readily accounted for, since it is difficult to determine the precise point at which the precipitate disappears. The resulting solution reacts very readily with excess tellurium to form a telluride containing a larger amount of tellurium and the tendency in general is to overrun the true end-point. So far as may be determined from the experimental data, the composition of the solution in equilibrium with the normal telluride does not vary appreciably with the concentration.

The composition of the solution in equilibrium with an excess of metallic tellurium may be obtained with considerable precision. Here, in general, the tendency will be to obtain a value which is too low, since the final endpoint is approached somewhat slowly and the influence of impurities, such as air and water vapor, is to remove sodium from the solution and thus yield too low a value for the amount of tellurium dissolved. It is evident from the table that the amount of tellurium dissolved at this stage of the reaction varies as a function of the concentration and that, as the concentration decreases, the amount of tellurium dissolved diminishes. At the higher concentrations, however, the composition of the solution varies only very little with the concentration and has a value corresponding very nearly with that of the formula $\mathrm{Na}_{2} \mathrm{Te}_{4}$.

The material left behind when a solution of a complex telluride is evaporated has a distinctly metallic appearance. It cannot be definitely stated that the resulting precipitate constitutes a homogeneous phase but, in any case, it is clear from the results of thermal analysis that free tellurium is not present under these conditions. We have, therefore, a very interesting system in which the normal sait is a non-metallic substance, while those compounds which contain larger amounts of the less electropositive element exhibit metallic properties in the solid state.

Since the normal telluride, $\mathrm{Na}_{2} \mathrm{Te}$, is only slightly soluble in liquid ammonia and since the composition of the solution in equilibrium with the normal telluride corresponds to the formula $\mathrm{Na}_{2} \mathrm{Te}_{2}$, it follows that in these solutions the initial complex telluride formed has a composition $\mathrm{Na}_{2} \mathrm{Te}_{2}$. Whether the final solution in equilibrium with metallic tellurium, which has a composition corresponding with that of the formula $\mathrm{Na}_{2} \mathrm{Te}_{4}$, actually consists chiefly of a complex of this composition or of a mixture of two or more complexes, having larger and smaller amounts of tellurium, remains uncertain. The nearness with which the composition of the more concentrated solutions approaches the value $\mathrm{Na}_{2} \mathrm{Te}_{4}$ inclines one to the view that the solution consists, for the most part, of a complex corresponding to this composition.

As regards the nature of these complex tellurides, it is clear that they do not differ materially from the corresponding complex sulfides which have 
been investigated in some detail by Küster and Heberlein. ${ }^{12}$ The phenomena in ammonia solutions are probably somewhat less complex than that in aqueous solutions, since in all likelihood hydrolysis does not take place to an appreciable extent in ammonia. This tendency of the electronegative elements of higher valence to form complex salts appears to be a very general one and certainly is not restricted to the elements of the sixth group. The behavior of the elements of the fifth group is very similar to that of the sixth, the chief difference being that the initial compounds formed are much less soluble in the case of these latter elements. Eventually, however, similar compounds are formed. The tendency of phosphorus to form complex phosphides is well known.

In the case of the elements of the fourth group, reaction takes place fairly readily with lead, but somewhat slowly with tin. What is striking in the case of the complexes formed between sodium and lead is the relative instability of the initial compound formed corresponding to the formula $\mathrm{Na}_{4} \mathrm{~Pb}$. This compound breaks down in solution into free metallic sodium and a complex richer in lead. The similarity of the reactions between the alkali metals and the less electropositive elements up to and including the fourth group of elements is unmistakable and clearly represents a fundamental property of these elements. In these compounds, the less electropositive constituent, which in many cases is a metallic substance in the elementary state, functions as anion. Apparently, all these elements are capable of acting as positive ions in the presence of less electropositive elements and as negative ions in the presence of more electropositive elements.

It is interesting to note that the complex salts of the most strongly electronegative elements, such as the iodides and sulfides, are non-metallic in the pure state. As the more electronegative elements become less electronegative, however, the resulting complexes become more and more metallic in their properties. Thus, while the normal telluride is non-metallic the complex tellurides are metallic, in the case of the fifth group of elements, the normal salts themselves are metallic, as for example, the normal antimonide, $\mathrm{Na}_{3} \mathrm{Sb}$. There is nothing to indicate that there is any fundamental change in the nature of the compounds as we pass from the iodides and sulfides through the tellurides to the antimonides and plumbides. It may be inferred, therefore, that, since the iodides and sulficles, as well as the normal tellurides are salt-like in character, the corresponding compounds of the less strongly electronegative elements are likewise salt-like in their properties; that is, they consist of oppositely charged ionic constituents. ${ }^{13}$

The precise nature of the complex tellurides can, of course, not be de-

12 Kïster and Heberlein, $Z$, anorg. Chem., 43, 53 (1905).

13 Compare Kraus, This JournaL, 44, 1216 (1922). 
termined without a knowledge of their complexity in solution. The complex present in solution in equilibrium with the normal telluride has a composition corresponding with that of the formula $\mathrm{Na}_{2} \mathrm{Te}_{2}$. In a subsequent paper it will be shown that the properties of the complex telluride correspond with this formula.

\section{Summary}

1. The reaction between sodium and tellurium in liquid ammonia has been studied and the composition of the resulting solutions has been determined under various conditions.

2. The initial compound formed is the normal telluride, corresponding to the formula $\mathrm{Na}_{2}{ }_{2} \mathrm{Te}$. In equilibrium with the normal telluride, the complex in solution has a composition corresponding to the formula $\mathrm{Na}_{2} \mathrm{Te}_{2}$. In equilibrium with free tellurium the solution has a composition which varies as a function of the concentration. The maximum amount of tellurium present corresponds approximately with the formula $\mathrm{Na}_{2} \mathrm{Te}_{4}$.

3. The significance of the results obtained is briefly discussed.

WORCESTER, MASSACHUSETTS

\section{NOTE}

The Stability of Phthalate Solutions as Standards in Hydrogen-ion Work.-Solutions of potassium hydrogen phthalate have long been used for the standardization of alkaline solutions. Clark ${ }^{1}$ more recently has recommended their use as standards in hydrogen-ion work. Their value for the latter use has been questioned by Oakes and Salisbury? who found that solutions of the most carefully purified salt gave a progressive decrease in hydrogen-ion concentration when in contact with the hydrogen electrode. Merrill ${ }^{3}$ also noted drifts in potential when phthalate solutions were used with the hydrogen electrode.

The potassium hydrogen phthalate used in our experiments was obtained from a manufacturer specializing in buffer salts for hydrogen-ion work and was not purified further by us. It was, however, tested by titration against sodium hydroxide solution with phenolphthalein as an indicator and found to give the theoretical value. A $0.2 M$ solution of the salt was prepared and from a portion of this solution another was made, as described by Clark, ${ }^{1}$ to give a Sörensen value $P_{\mathrm{H}}$ 5.6. The hydrogen-electrode potentials of these solutions were obtained with a Bunker hydrogen electrode, a Leeds and Northrup Type " $\mathrm{K}$ " potentiometer and a Weston cell calibrated by the Bureau of Standards.

"Clark, "The Determination of Hydrogen Ions," Williams and Wilkins Co., Baltimore, 1920.

${ }^{2}$ Oakes and Salisbury, This Journal, 44, 948 (1922).

'Merrill, itid., 43, 2688 (1921). 